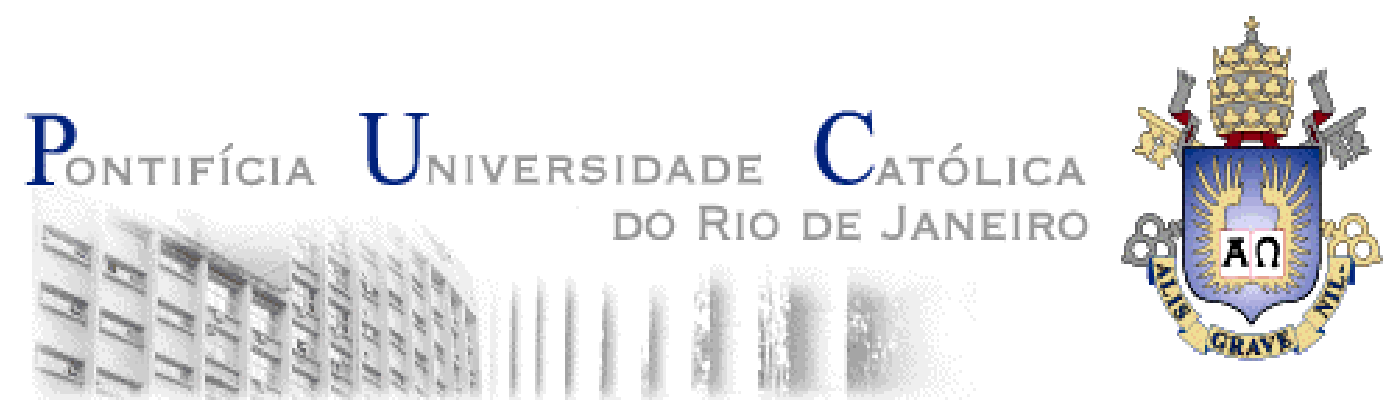

Luis Fernando Palomino Yupa

Estudo Experimental da Deposição de Parafina em Escoamento Turbulento

Dissertação de Mestrado

Dissertação apresentada como requisito parcial para obtenção do título de Mestre pelo Programa de Pós-Graduação em Engenharia Mecânica da PUC-Rio.

Orientador: Prof. Luis Fernando Alzuguir Azevedo

Rio de Janeiro

Setembro de 2010 


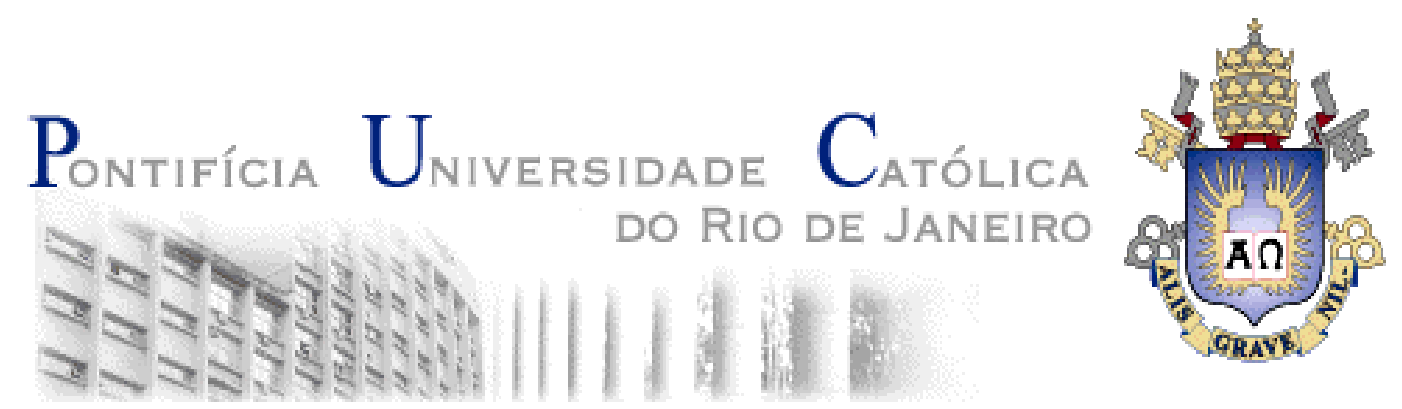

Luis Fernando Palomino Yupa

\section{Estudo Experimental da Deposição de Parafina em Escoamento Turbulento}

Dissertação apresentada como requisito parcial para obtenção do título de Mestre pelo Programa de PósGraduação em Engenharia Mecânica da PUC-Rio. Aprovada pela Comissão Examinadora abaixo assinada.

Prof. Luis Fernando Alzuguir Azevedo Orientador

Departamento de Engenharia Mecânica - PUC-Rio

Prof. Paulo Roberto de Souza Mendes Departamento de Engenharia Mecânica - PUC-Rio

Prof. Sergio Leal Braga Departamento de Engenharia Mecânica - PUC-Rio

Prof. José Eugenio Leal Coordenador Setorial do Centro

Técnico Cientifico - PUC-Rio

Rio de Janeiro, 21 de Setembro de 2010 
Todos os direitos reservados. É proibida a reprodução total ou parcial do trabalho sem autorização da universidade, do autor e do orientador.

\section{Luis Fernando Palomino Yupa}

Graduou-se em Engenharia Mecânica no Dpto. de Engenharia Mecânica da UNSA (Universidad Nacional de San Agustin, Arequipa-Peru), em 2008.

Ficha Catalográfica

Palomino Yupa, Luis Fernando
Estudo experimental da deposição de parafina
em escoamento turbulento / Luis Fernando Palomino
Yupa ; orientador: Luiz Fernando Alzuguir Azevedo. -
2010. $\quad 100$ f.: il.(color.); 30 cm
Dissertação (mestrado)-Pontifícia Universidade
do Rio de Janeiro, Departamento de
Engenharia Mecânica, 2010.
Inclui bibliografia
1. Engenharia mecânica - Teses. 2.
Deposição de parafina. 3. Dutos. 4. Escoamento
turbulento. I. Azevedo, Luis Fernando Alzuguir. II.
$\begin{aligned} & \text { Pontifícia Universidade Católica do Rio de Janeiro. } \\ & \text { Departamento de Engenharia Mecânica. III. Título. }\end{aligned}$

CDD: 621 
"Tudo posso naquele que me fortalece"

Filipense 4:13 


\section{Agradecimentos}

A Deus por cada novo dia.

A minha mãe, pai e irmã por seu amor e apoio incondicional.

A meu professor Luis Fernando Alzuguir Azevedo, meu orientador, pela amizade e enorme paciência que teve comigo durante estes anos.

A meus amigos: Helena, Paula, Leo, Fabio, Guillermo, Bruno, Carlos, Jose, Marco, Darwin pela amizade e pelos bons momentos compartilhados nestes anos.

Agradecimentos em particular ao $\mathrm{CNPq}$, e à PUC-Rio, pelo apoio financeiro fornecido, sem o qual este trabalho simplesmente não teria sido possível. 


\section{Resumo}

Palomino Yupa, Luis Fernando; Azevedo, Luis Fernando Alzuguir. Estudo Experimental da Deposição de Parfina em Escoamento Turbulento. Rio de Janeiro, 2010. 100p. Dissertação de Mestrado - Departamento de Engenharia Mecânica, Pontifícia Universidade Católico do Rio de Janeiro.

Um dos principais problemas encontrados na produção de petróleo em águas profundas é a deposição de parafina em linhas de produção e transporte. À medida que o óleo escoa pelos dutos, perde calor para o ambiente marinho mais frio, reduzindo a solubilidade dos hidrocarbonetos mais pesados, podendo levar à deposição destes componentes nas paredes do duto. A deposição de parafina pode causar redução da capacidade de produção e até bloqueio total da linha. A capacidade de previsão da variação temporal destes depósitos, assim como a sua distribuição espacial ao longo do duto, são informações relevantes para o projeto e a operação das linhas. Uma revisão atualizada da literatura revela que os mecanismos responsáveis pela deposição de parafina ainda não são bem compreendidos. O presente trabalho faz parte de um programa de pesquisa em andamento voltado para o estudo dos mecanismos de deposição de parafina em dutos. Foram conduzidos experimentos de laboratório onde soluções de parafina e querosene com propriedades conhecidas escoavam por seção de testes especialmente projetada para permitir a medição da variação espacial e temporal da espessura dos depósitos. As medições foram realizadas para regime de escoamento turbulento utilizando um microscópio óptico. Os resultados obtidos mostraram que a espessura de depósito decresce com o aumento do número de Reynolds, sendo inferior aos valores obtidos para a condição de regime laminar. A boa qualidade das visualizações obtidas permitiu observar a remoção de depósito por cisalhamento. Os resultados obtidos estão disponíveis para validar soluções numéricas e auxiliar no entendimento dos mecanismos básicos responsáveis pela deposição de parafina.

\section{Palavras-chave}

Deposição de parafina; dutos; escoamento turbulento. 


\section{Abstract}

Palomino Yupa, Luis Fernando; Azevedo, Luis Fernando Alzuguir. Experimental Study Wax Deposition in Turbulent Flow. Rio de Janeiro, 2010. 100p. MSc. Dissertation - Departamento de Engenharia Mecânica, Pontificia Universidade Católica do Rio de Janeiro.

One of the main problems encountered in oil production in deepwaters is the wax deposition in production and transportation lines. As the oil flows in the pipelines, it loses heat to the colder outside environment, reducing its solubility to heavy hydrocarbons, and potentially leading to deposition at the pipe walls. Wax deposition may cause loss of production and even the complete blockage of the line. The ability to predict the spatial and temporal variation of the deposits is relevant to the design and operation of subsea lines. An updated literature review revealed that the mechanisms responsible for wax deposition are still not fully understood. The present work is part of an ongoing research project aimed at studying the mechanisms responsible for wax deposition in pipelines. Laboratory experiments were conducted for turbulent flow of wax and querosene solutions through a specially designed test section that allowed for optical acces. An optical microscope was employed for measuring the wax deposit thicknesses which were shown to decrease with increasing Reynolds number. The deposits measured in the turbulent regime were smaller than those for the laminar regime. The good quality of the visualizations obtained allowed the observation of wax deposit removal by shear effects. The experimental results obtained are available for comparison with numerical solutions, what can contribute to the study of the basic mechanisms responsible for wax deposition.

\section{Keywords}

Wax Deposition; turbulent flow; pipelines. 


\section{Sumário}

1. Introdução

2. Revisão Bibliográfica 23

2.1. Caracteristicas da parafina 23

2.2. Temperatura de aparecimento de Cristais (TIAC) 26

2.3. Mecanismos de Deposição 27

2.3.1. Difusão molecular 28

2.3.2. Difusão Browniana 29

2.3.3. Dispersão por Cisalhamento 30

2.3.4. Deposição por Efeitos Gravitacionais 32

2.4. Taxa de Deposição de Parafina 33

2.5. Erosão do Deposito de Parafina por Cisalhamento 35

2.6. Adesão da Camada Depositada. 36

2.7. Envelhecimento e Endurecimento do Deposito 36

2.8. Resultados Relevantes da Bibliografia. 38

3. Procedimento e Montagem Experimental 39

3.1. Montagem experimental 39

3.1.1. Controle e Medição da Temperatura 46

3.1.2. Solução de Parafina 48

3.1.3. Sistema de visualização e processamento de imagens 48

3.2. Procedimento experimental 50

4. Resultados 53

4.1. Resultados de Perfis de Temperatura 53

4.2. Ensaios Preliminares de Deposição de Parafina 58

4.2.1. Calibração das Imagens 58

4.2.2. Determinação do Tempo para Obtenção de Regime Permanente 59

4.2.3. Testes de Simetria do Depósito de Parafina 60

4.2.4. Avaliação da Movimentação das Paredes do Canal 62 
4.3. Visualização da Deposição de Parafina sob Escoamento Laminar e Turbulento

4.4. Resultados Quantitativos para a Deposição de Parafina 79

4.4.1. Espessuras de Depósito de Parafina para Regime Permanente 79

4.4.2. Evolução Espacial e Temporal dos Depósitos de Parafina 82

5 Conclusões 91

6 Referências Bibliográficas 93

$\begin{array}{ll}\text { Apêndice } & 97\end{array}$

Apêndice A.1 98

Apêndice A.2 99

Apêndice A.3 100 


\section{Lista de Figuras}

Figura 1.1: Disposição típica de linhas submarinas de

transporte de óleo 19

Figura 1.2: Linha parcialmente bloqueada por

deposição de parafina (Cortesia do CENPES/Petrobras) 20

Figura 1.3: Operação de remoção de parafina na plataforma PXA $-3 \ldots .21$

Figura 2.1: Variação da temperatura de solidificação de

parafinas normais versus número de átomos de carbono 25

Figura 2.2: Perfil de concentração de cristais de parafina precipitados [Burger et al, 1981] 30

Figura 2.3: (a) Distribuição de partículas na seção

transversal do duto (b) histograma de probabilidade, para $\mathrm{Re}=67$ 32

Figura 2.4: (a) Distribuição de partículas na seção transversal

do duto com (b) histograma de probabilidade, para $\mathrm{Re}=350$.

Figura 2.5: Vista da estrutura do deposito imóvel

Figura 3.1: Disposição esquemática da seção de teste 40

Figura 3.2: llustração da seção retangular composta de dois blocos de cobre laterais

Figura 3.3: Vista isométrica de acrílico e as paredes de cobre no inicio .. 41 Figura 3.4: Vista isométrica de acrílico e as paredes de cobre no final.... 41

Figura 3.5: Desenho da peça de acrílico na saída do canal..................... 42

Figura 3.6: Desenho da peça de acrílico na entrada do canal ................. 42

Figura 3.7: Vista isométrica e lateral do reservatório de armazenamento 43

Figura 3.8: Vista do topo e tampa do reservatório de armazenamento .... 44

Figura 3.9: Imagem frontal da seção de teste ....................................... 45

Figura 3.10: Imagem lateral da seção de teste .......................................46

Figura 3.11: Diagrama da localização dos termopares ........................... 47

Figura 3.12: Imagem da disposição dos termopares na seção de teste .. 47

Figura 3.13: Imagem da mistura parafina-querosene............................. 48

Figura 3.14: Microscópio posicionado sob a seção de teste .................... 49 
Figura 3.15: Processamento com o programa de computador

Axiovision Rel 4.4.da Zeiss

Figura 3.16: llustração das posições de visualização das espessuras de depósito ao longo do canal. 52

Figura 4.1: Evolução temporal do perfil axial de temperatura da parede interna do canal, para regime laminar com $R e=1660$ 55

Figura 4.2: Teste de repetibilidade do processo de resfriamento da parede do canal. Resultados de 17 replicações dos experimentos para perfis de temperatura após 2 minutos do início do resfriamento. Regime de escoamento laminar, $R e=1660 \ldots 57$ Figura 4.3: Comparação dos perfis de temperatura ao longo da parede do canal após 2 minutos do início do resfriamento para escoamento laminar $(R e=1660)$ e turbulento $(R e=6900)$. Barras verticais representam o espalhamento obtido na replicação de 17 experimentos. .57

Figura 4.4: Variação temporal da espessura do depósito de parafina para uma posição axial no meio do canal para diferentes valores do número de Reynolds. Teste para a determinação do tempo necessário para a obtenção da condição de regime permanente na espessura do depósito de parafina

Figura 4.5: Testes para a verificação da simetria da distribuição de temperatura e espessura de depósito ao longo do canal. Resultados de regime permanente para $R e=5650, T f=40^{\circ} \mathrm{C}$ e $T p=15^{\circ} \mathrm{C}$.

Figura 4.6: Testes para a verificação da simetria dos depósitos de parafina. Resultados da obtidos para a condição de regime permanente, com $R e=5650$, temperatura de entrada $T e=40^{\circ} \mathrm{C}$ e temperatura das paredes, $T p=15^{\circ} \mathrm{C}$ 62

Figura 4.7: Deslocamentos transversais da parede de cobre devido ao resfriamento da parede como função do tempo contado do início do resfriamento. Teste para regime laminar com $R e=1660$, para três posições longitudinais do canal: início, meio e fim do canal. Temperatura de entrada do querosene a $40^{\circ} \mathrm{C}$ e temperatura final de resfriamento da parede igual a $15^{\circ} \mathrm{C}$ 
Figura 4.8: Vista esquemática das posições das regiões de observação com microscópio ao longo do canal (reprodução da Figura 3.16)

Figura 4.9: Visão esquemática da seção transversal do canal indicando a formação de depósito de parafina nas faces internas das paredes de vidro..

Figura 4.10: Região de concentração de cristais perto da parede nos primeiros segundos depois de iniciado o resfriamento

Figura 4.11: Seqüência de imagens obtidas na região de entrada do canal, mostrando a evolução temporal e espacial do depósito para o teste com $\mathrm{Te}=40^{\circ} \mathrm{C}, \mathrm{Tpi}=15^{\circ} \mathrm{C}$ e $\mathrm{Re}=1660$. $O$ aumento utilizado foi 32x. As imagens fora obtidas nas posições axiais 1,2 e 3, de acordo com a definição da Figura 4.8. As figuras correspondem aos instantes de tempo, 0, 2, 5, 10, 30 e 150 minutos, contados após o início do resfriamento. 69

Figura 4.12: Seqüência de imagens obtidas na região de saída do canal, mostrando a evolução temporal e espacial do depósito para o teste com $\mathrm{Te}=40^{\circ} \mathrm{C}, \mathrm{Tpi}=15^{\circ} \mathrm{C}$ e $\mathrm{Re}=1660$. $O$ aumento utilizado foi 32x. As imagens fora obtidas nas posições axiais 22, 23 e 24, de acordo com a definição da Figura 4.8. As figuras correspondem aos instantes de tempo, 0, 2, 5, 10, 30 e 150 minutos, contados após o início do resfriamento.. .72

Figura 4.13: Seqüência de imagens obtidas na região de entrada do canal, mostrando a evolução temporal do depósito para o teste com $\mathrm{Te}=40^{\circ} \mathrm{C}, \mathrm{Tpi}=15^{\circ} \mathrm{C}$ e $\mathrm{Re}=6900$. $\mathrm{O}$ aumento utilizado foi 32x. As imagens fora obtidas nas posições axiais 1, 2 e 3, de acordo com a definição da Figura 4.8. As figuras correspondem aos instantes de tempo, 0, 2, 5, 10, 30 e 150 minutos, contados após o início do resfriamento. 74

Figura 4.14: Seqüência de imagens obtidas na região de saída do canal, mostrando a evolução temporal do depósito para o teste com $\mathrm{Te}=40^{\circ} \mathrm{C}, \mathrm{Tpi}=15^{\circ} \mathrm{C}$ e $\mathrm{Re}=6900$. $O$ aumento utilizado foi 50x. As imagens fora obtidas nas posições axiais 22, 23 e 24, de acordo com a definição da Figura 4.8. As figuras correspondem aos instantes de tempo, 0, 2, 5, 10, 30 e 150 minutos, contados 
após o início do resfriamento.

Figura 4.15: Seqüência de imagens obtidas na metade do canal, posição 16 , mostrando a evolução temporal do depósito para o teste com $\mathrm{Te}=40^{\circ} \mathrm{C}, \mathrm{Tpi}=15^{\circ} \mathrm{C}$ e $\mathrm{Re}=1660$. Aumento utilizado de 32x.

(a) 0 minutos, (b) 2 minutos, (c) 4 minutos, (d) 6 minutos,

(e) 8 minutos, (f) 10 minutos, (g) 12 minutos, e (h) 14 minutos

Figura 4.16: Seqüência de imagens obtidas na metade do canal, posição 16 , mostrando a evolução temporal do depósito para o teste com $\mathrm{Te}=40^{\circ} \mathrm{C}, \mathrm{Tpi}=15^{\circ} \mathrm{C}$ e $\mathrm{Re}=6900$. O aumento utilizado foi 50x. (a) 0 minuto, (b) 2 minutos, (c) 4 minutos, (d) 6 minutos, (e) 8 minutos, (f) 10 minutos, (g) 12 minutos, e (h) 14 minutos 78

Figura 4.17: Distribuição espacial da espessura de depósito de parafina para regime permanente (150 min.) e diferentes números de Reynolds. Resultados para $\mathrm{Te}=40^{\circ} \mathrm{C}, \mathrm{Tp}=15^{\circ} \mathrm{C}$ e $\mathrm{TIAC}=28,7^{\circ} \mathrm{C} \ldots \ldots .81$ Figura 4.18: Evolução temporal e espacial da espessura do depósito de parafina na parede interior do canal sob escoamento laminar para $\mathrm{Re}=1660$. Resultados para $\mathrm{Te}=40^{\circ} \mathrm{C}, \mathrm{Tp}=15^{\circ} \mathrm{C}$ e $\mathrm{TIAC}=28,7^{\circ} \mathrm{C} \ldots 83$ Figura 4.19: Distribuição axial da temperatura ao longo da parede de cobre para Reynolds igual 1660, para os instantes iguais a 0, 2, $5,10,30$ e $150 \mathrm{~min}$. Resultados para $\mathrm{Te}=40^{\circ} \mathrm{C}$. $\mathrm{Tp}=15^{\circ} \mathrm{C}$. .84

Figura 4.20: Evolução temporal e espacial da espessura do depósito de parafina na parede interior do canal sob escoamento turbulento para Reynolds igual 4400. Resultados para $\mathrm{Te}=40^{\circ} \mathrm{C}$. $\mathrm{Tp}=15^{\circ} \mathrm{C}$ .85

Figura 4.21: Distribuição axial da temperatura ao longo da parede de cobre para Reynolds igual 4400 para os instantes iguais a 0, 2, 5, 10, 30 e 150 min após do inicio da experiência. Resultados para $\mathrm{Te}=40^{\circ} \mathrm{C}$. $\mathrm{Tp}=15^{\circ} \mathrm{C}$ .86

Figura 4.22: Evolução temporal e espacial da espessura do depósito de parafina na parede interior do canal sob escoamento turbulento para Reynolds igual 5650. Resultados para $\mathrm{Te}=40^{\circ} \mathrm{C}$. $\mathrm{Tp}=15^{\circ} \mathrm{C}$

Figura 4.23: Distribuição axial da temperatura ao longo da parede de cobre para Reynolds igual 5650 para os instantes iguais a 0,2 , 5, 10, 30 e 150 minutos após do inicio da experiência.

Resultados para $\mathrm{Te}=40^{\circ} \mathrm{C}$. $\mathrm{Tp}=15^{\circ} \mathrm{C}$ 
Figura 4.24: Evolução temporal e espacial da espessura do depósito de parafina na parede interior do canal sob escoamento turbulento para Reynolds igual 6900.

Resultados para $\mathrm{Te}=40^{\circ} \mathrm{C}$. $\mathrm{Tp}=15^{\circ} \mathrm{C}$ 89

Figura 4.25: Distribuição axial da temperatura ao longo da parede de cobre para Reynolds igual 6900 para os instantes iguais a 0, 2, 5, 10, 30 e 150 minutos após do inicio da experiência.

Resultados para $\mathrm{Te}=40^{\circ} \mathrm{C}$ e $\mathrm{Tp}=15^{\circ} \mathrm{C}$ 89

Figura 4.26: Taxa de crescimento de depósito para uma posição no meio do canal, como função do número de Reynolds.

Resultados para os 10 primeiros minutos dos testes 90

Figura 4.27: Taxa de crescimento de depósito para uma posição no meio do canal, como função do número de Reynolds.

Resultados para os 30 primeiros minutos dos testes. 


\section{Lista de tabelas}

Tabela 2.1 - Características físicas de algumas parafinas

normalmente encontradas no petróleo.... Erro! Indicador não definido.24

Tabela 2.2 - Comparação entre diferentes técnicas

na determinação de TIAC. Plasencia et al. [20] - Reprodução parcial. ...27 


\section{Lista de símbolos}

$\begin{array}{lll}A & \text { área } & {\left[\mathrm{m}^{2}\right]} \\ C & \text { fração volumétrica da parafina na solução } & {[-]} \\ c_{p} & \text { fração de partículas solidas } & {[-]} \\ c_{v} & \text { coeficiente de difusão } & {\left[\mathrm{m}^{2} / \mathrm{s}\right]} \\ \bar{c}_{r} & \text { constante da taxa de deposição } & {\left[\mathrm{kg} / \mathrm{m}^{2}\right]} \\ \mathrm{m} & \text { massa } & {[\mathrm{kg}]} \\ r & \text { coordenada radial } & {[\mathrm{m}]} \\ \mathrm{R} & \text { raio do duto } & {[\mathrm{m}]} \\ \operatorname{Re} & \text { Número de Reynolds } & {[-]} \\ t & \text { tempo } & {[\mathrm{s}]} \\ T & \text { temperatura } & {\left[{ }^{\circ} \mathrm{C}\right]}\end{array}$




\section{Símbolos gregos}

$\begin{array}{lll}\Delta T & \text { diferença de temperatura } & {\left[{ }^{\circ} \mathrm{C} ; \mathrm{K}\right]} \\ \Delta P & \text { queda de pressão } & {[\mathrm{kPa}]} \\ \mu & \text { viscosidade } & {\left[\mathrm{N} \mathrm{s} \mathrm{m}{ }^{-2}\right]} \\ \rho & \text { massa especifica } & {\left[\mathrm{kg} \cdot \mathrm{m}^{-3}\right]} \\ \nu & \text { taxa de cisalhamento na parede. } & {\left[\mathrm{s}^{-1}\right]}\end{array}$




\section{Subscritos}

$\begin{array}{ll}\text { amb } & \text { ambiente } \\ e & \text { entrada no canal } \\ p & \text { parede } \\ B & \text { Browniana }\end{array}$

